\title{
Binational Dengue Outbreak Along the United States-Mexico Border - Yuma County, Arizona, and Sonora, Mexico, 2014
}

\begin{abstract}
Jefferson M. Jones, $\mathrm{MD}^{1,2}$; Benito Lopez, $\mathrm{MD}^{3}$; Laura Adams, DVM ${ }^{1,4}$; Francisco Javier Navarro Gálvez, MD ${ }^{5}$; Alfredo Sánchez Núñez, MD ${ }^{6}$; Nubia Astrid Hernández Santillán, MD ${ }^{5}$; Lydia Plante, $\mathrm{MPH}^{1}$; Ryan R. Hemme, PhD ${ }^{7}$; Mariana Casal, MD ${ }^{1}$; Elizabeth A. Hunsperger, PhD ${ }^{7}$; Jorge Muñoz-Jordan, $\mathrm{PhD}^{7}$; Veronica Acevedo ${ }^{7}$; Kacey Ernst, $\mathrm{PhD}^{8}$; Mary Hayden, $\mathrm{PhD}^{9}$; Steve Waterman, $\mathrm{MD}^{10}$; Diana Gomez, MPH${ }^{3}$; Tyler M. Sharp, $\mathrm{PhD}^{7}$; Kenneth K. Komatsu, $\mathrm{MPH}^{1}$; Dengue Investigative Team
\end{abstract}

Dengue is an acute febrile illness caused by any of four dengue virus types (DENV-1-4). DENVs are transmitted by mosquitos of the genus Aedes ( 1 ) and are endemic throughout the tropics (2). In 2010, an estimated 390 million DENV infections occurred worldwide (2). During 2007-2013, a total of three to 10 dengue cases were reported annually in Arizona and all were travel-associated. During September-December 2014, coincident with a dengue outbreak in Sonora, Mexico, 93 travel-associated dengue cases were reported in Arizona residents; 70 (75\%) cases were among residents of Yuma County, which borders San Luis Río Colorado, Sonora, Mexico. San Luis Río Colorado reported its first case of locally acquired dengue in September 2014. To investigate the temporal relationship of the dengue outbreaks in Yuma County and San Luis Río Colorado and compare patient characteristics and signs and symptoms, passive surveillance data from both locations were analyzed. In addition, household-based cluster investigations were conducted near the residences of reported dengue cases in Yuma County to identify unreported cases and assess risk for local transmission. Surveillance data identified 52 locally acquired cases (21\% hospitalized) in San Luis Río Colorado and 70 travel-associated cases (66\% hospitalized) in Yuma County with illness onset during September-December 2014. Among 194 persons who participated in the cluster investigations in Yuma County, 152 (78\%) traveled to Mexico at least monthly during the preceding 3 months. Four (2\%) of 161 Yuma County residents who provided serum samples for diagnostic testing during cluster investigations had detectable DENV immunoglobulin M (IgM); one reported a recent febrile illness, and all four had traveled to Mexico during the preceding 3 months. Entomologic assessments among 105 households revealed 24 water containers per 100 houses colonized by Ae. aegypti. Frequent travel to Mexico and Ae. aegypti colonization indicate risk for local transmission of DENV in Yuma County. Public health officials in Sonora and Arizona should continue to collaborate on dengue surveillance and educate the public regarding mosquito abatement and avoidance practices. Clinicians evaluating patients from the U.S.-Mexico border region should consider dengue in patients with acute febrile illness and report suspected cases to public health authorities.

In areas of Mexico where dengue is endemic, approximately $30 \%$ of patients with suspected dengue are tested by enzyme-linked immunosorbent assay (ELISA) to detect nonstructural protein 1 (NS1) or DENV IgM or immunoglobulin G; approximately $10 \%$ of NS1-positive specimens are further tested by reverse transcription-polymerase chain reaction (RT-PCR) to identify the infecting DENV (3). Symptomatic patients who are not tested are classified as probable cases. Disease severity is classified according to 1997 World Health Organization (WHO) dengue case definitions (4).

In Arizona, suspected dengue cases are reported by health care providers and commercial laboratories. When possible, specimens from suspected dengue cases at commercial laboratories are forwarded to CDC Dengue Branch for confirmatory testing by real time RT-PCR (rRT-PCR) for detection and typing of DENV, and by anti-DENV IgM ELISA.

Passive surveillance data from Yuma County and San Luis Rio Colorado were reviewed, and laboratory-confirmed cases from Yuma County and San Luis Rio Colorado were analyzed. Available medical records of patients in Yuma County with symptom onset during September-December 2014 were abstracted, using a modification of CDC's Dengue Case Investigation Form.* Clinical case classifications were assigned using both the 1997 (4) and 2009 WHO dengue case definitions (1).

During December 15-19, 2014, and January 15-16, 2015, household-based cluster investigations were conducted in Yuma County by public health workers from Yuma County Department of Public Health, Arizona Department of Health Services, University of Arizona, and CDC. Households within a 50-meter radius of residences of persons with laboratoryconfirmed dengue were visited within 90 days of the patient's reported illness onset. Among households where at least one adult agreed to participate, individual and household questionnaires were administered in English or Spanish to collect information on demographic, medical, and behavioral characteristics. Blood specimens were collected and sera were tested by rRT-PCR and anti-DENV IgM ELISA. Entomologic assessments of the house and yard were conducted to identify

\footnotetext{
*Variables added to the CDC's Dengue Case Investigation Form included admission to the intensive care unit (ICU), length of stay in ICU, and if care was received in Mexico. Variables removed included length of residency in current city, history of yellow fever vaccine, and history of previous dengue diagnosis. http://www.cdc.gov/dengue/resources/dengueCaseReports/DCIF_English.pdf.
} 
potential and colonized mosquito breeding sites. Data were translated into Breteau, container, and house indices (established indicators of mosquito density) (5).

During September-November 2014, a total of 52 laboratoryconfirmed dengue cases were identified in San Luis Río Colorado, Sonora: 30 (58\%) by detection of NS1, 21 (40\%) by detection of DENV IgM, and $1(2 \%)$ by NS1 and RT-PCR. Thirty-two (62\%) patients were female; the median age was 34.5 years (range $=0-76$ years). Symptom onset dates ranged from September 20-November 13 (Figure). The most commonly reported signs and symptoms were fever (100\%), headache (98\%), arthralgia (92\%), and myalgia (90\%) (Table 1). Three $(6 \%)$ patients met the case definition for dengue hemorrhagic fever ${ }^{\dagger}$ (DHF), and $11(21 \%)$ were hospitalized; there were no deaths.

\footnotetext{
$\dagger$ Dengue hemorrhagic fever is characterized by all of the following: fever lasting 2-7 days, evidence of hemorrhagic manifestation or a positive tourniquet test, thrombocytopenia (platelets $\leq 100,000 / \mu \mathrm{L}$ ), and evidence of plasma leakage indicated by hemoconcentration (an increase in hematocrit $\geq 20 \%$ above average for age or a decrease in hematocrit $\geq 20 \%$ of baseline after fluid replacement therapy), or pleural effusion, or ascites or hypoproteinemia. http://www.cdc. gov/dengue/clinicalLab/caseDef.html.
}

In Yuma County, 70 laboratory-confirmed cases were identified during October 18-December 5. Eight (11\%) were positive by rRT-PCR alone, $48(69 \%)$ by IgM ELISA alone, and $14(20 \%)$ by both rRT-PCR and IgM ELISA. Forty-two $(60 \%)$ patients were female, the median age was 48 years (range $=1-87$ years), and the most commonly reported symptoms were fever $(87 \%)$, myalgia $(61 \%)$, headache $(61 \%)$, and rash (40\%). No patients met the case definition for DHF; 37 (53\%) were hospitalized, and none died. Travel history was available for $60(86 \%)$ patients, and all reported travel to Mexico $<14$ days before illness onset. DENV-1 was the only DENV detected by RT-PCR from patients in San Luis Rio Colorado and Yuma County.

In Yuma County, 39 household-based cluster investigations were conducted (median number of households/cluster investigation $=3$; range $=1-6)$. Among 351 eligible houses, $55(16 \%)$ heads-of-household refused; 162 (46\%) houses were occupied, but no residents were present; and $21(6 \%)$ houses appeared vacant, leaving 113 (32\%) participating households. Among the 113 responding heads-of-household, 50 (44\%) reported lacking screens on some or all household windows; 44 (39\%)

FIGURE. Number of laboratory-confirmed dengue cases, by week of symptom onset - San Luis Río Colorado, Sonora, Mexico, and Yuma County, Arizona, 2014

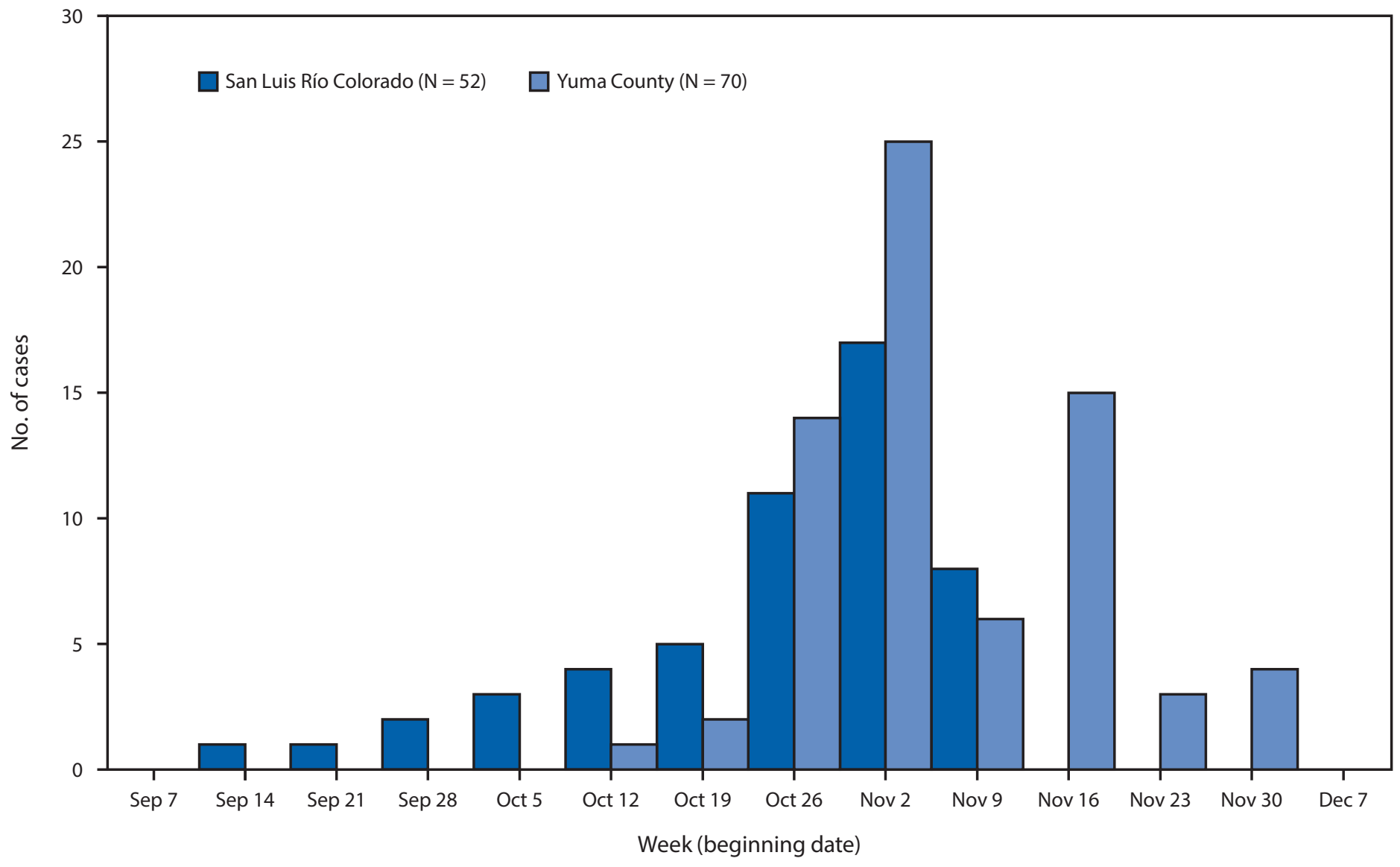


TABLE 1. Demographic characteristics and reported signs and symptoms of laboratory-confirmed dengue cases - Yuma County, Arizona, and San Luis Rio Colorado, Sonoma, Mexico, OctoberDecember 2014

\begin{tabular}{|c|c|c|}
\hline & $\begin{array}{l}\text { San Luis Río Colorado } \\
(\mathrm{n}=52) \\
\end{array}$ & $\begin{array}{c}\text { Yuma County } \\
(\mathrm{n}=70)\end{array}$ \\
\hline Characteristic & No. (\%) & No. (\%) \\
\hline Female & $32(62)$ & $42(60)$ \\
\hline Hospitalized & $11(21)$ & $37(53)$ \\
\hline \multicolumn{3}{|l|}{ Signs and symptoms } \\
\hline Fever & $52(100)$ & $61(87)$ \\
\hline Headache & $51(98)$ & $43(61)$ \\
\hline Myalgia & $47(90)$ & $43(61)$ \\
\hline Arthralgia & $48(92)$ & $25(36)$ \\
\hline Eye pain & $39(75)$ & $16(23)$ \\
\hline Rash & $21(40)$ & $28(40)$ \\
\hline Persistent vomiting & $3(6)$ & $14(20)$ \\
\hline Abdominal pain & $3(6)$ & $23(33)$ \\
\hline Ascites or pleural effusion & $0(0)$ & $1(1)$ \\
\hline \multicolumn{3}{|l|}{ Bleeding manifestations } \\
\hline Major* & $3(6)$ & $1(1)$ \\
\hline Minor $^{\dagger}$ & $7(13)$ & $14(20)$ \\
\hline Nausea or vomiting & $N A^{\S}$ & $40(57)$ \\
\hline Diarrhea & $N A^{\S}$ & $25(36)$ \\
\hline Anorexia & $N A^{\S}$ & $15(21)$ \\
\hline Lethargy & $N A^{\S}$ & $15(21)$ \\
\hline Cough & $N A^{\S}$ & $12(17)$ \\
\hline Hepatomegaly & $N A^{\S}$ & $1(1)$ \\
\hline Thrombocytopenia? & $N A^{\S}$ & $34(49)$ \\
\hline Low serum albumin ${ }^{* *}$ & $N A^{\S}$ & $16(23)$ \\
\hline Low serum protein $* *,+\dagger$ & $N A^{\S}$ & $8(11)$ \\
\hline \multicolumn{3}{|l|}{ Clinical case definition } \\
\hline Dengue fever & $49(94)$ & $51(70)$ \\
\hline 1997 WHO case definitions & & \\
\hline Dengue hemorrhagic fever & $3(6)$ & $0(0)$ \\
\hline \multicolumn{3}{|l|}{2009 WHO case definitions } \\
\hline Dengue fever & $N A^{\S}$ & $18^{+\dagger}(26)$ \\
\hline Dengue with warning signs & $N A^{\S}$ & $28(40)$ \\
\hline Severe dengue & $N A^{\S}$ & $5(7)$ \\
\hline
\end{tabular}

Abbreviations: $\mathrm{NA}=$ not available; $\mathrm{WHO}=$ World Health Organization.

* Purpura or ecchymosis (two persons); hematemesis (two persons).

† Petechiae, bleeding gums, epistaxis, unspecified mucosal bleeding, and hematuria.

$\S$ These variables not collected by passive surveillance used by Sonora Department of Public Health.

I $\leq 100,000$ platelets $/ \mu \mathrm{L}$.

** Lower than age-specified values.

${ }^{\dagger+}$ Nineteen (27\%) cases did not meet case definition of dengue fever based on available medical records (nine cases, fever not documented; 10 cases, $\geq 2$ additional compatible symptoms not documented).

reported leaving windows open during the night or day; and 97 (86\%) reported using air conditioning (Table 2). Among 447 residents of the participating households, 194 (43\%) responded to an individual questionnaire (median $=1$ person/ household; range $=1-6)$ and $253(57 \%)$ refused or were absent. Median age of participants was 40 years (range $=1-86$ years); median duration of residence in Arizona was 14.5 years (range $=1$ month-72 years). During the 3 months before interview, $152(78 \%)$ participants reported having traveled to Mexico at least once a month and $32(16 \%)$ reported using mosquito repellent (Table 2). Among 161 participants without
TABLE 2. Individual participant and household characteristics from household-based cluster investigations of dengue - Yuma County, Arizona, December 2014-January 2015

\begin{tabular}{lr}
\hline Individual characteristic $(\mathbf{n}=\mathbf{1 9 4}$ persons) & No. (\%) \\
\hline Female & $115(59)$ \\
Use mosquito repellent* & $32(16)$ \\
Travel to Mexico* & \\
Daily & $5(3)$ \\
Weekly & $99(51)$ \\
Monthly & $48(25)$ \\
Yearly & $3(2)$ \\
No travel or unknown & $39(20)$ \\
Survey conducted in Spanish & $129(66)$ \\
Febrile illness* & $56(29)$ \\
Method of payment for medical care & \\
Medicaid & $113(58)$ \\
Medicare & $15(8)$ \\
Other insurance & $27(14)$ \\
Care in Mexico & $24(12)$ \\
No access & $11(6)$ \\
Other or missing & $4(2)$ \\
Household characteristics $(\mathbf{n}=113$ households) & \\
Water source is public piping* & $109(96)$ \\
Store water in open container* & $11(10)$ \\
Visitors from outside United States* & $45(40)$ \\
Febrile illness in household* & $51(45)$ \\
Screens on some windows* & $25(22)$ \\
Screens on all windows* & $63(56)$ \\
Leave windows open* & $44(39)$ \\
Use air conditioning* & $97(86)$ \\
Mosquitoes observed inside home* & $42(37)$ \\
Sprayed or used other method to control mosquitoes* & $42(37)$ \\
Have septic tank & $10(9)$ \\
\hline * During the preceding 3 months. &
\end{tabular}

* During the preceding 3 months.

a previous dengue diagnosis who provided samples for testing, four (2\%) had detectable DENV IgM, indicating recent infection. All had traveled to Mexico during the preceding 3 months, and one reported a recent febrile illness.

Entomologic assessments in 105 households revealed 24 Ae aegypti colonized containers/100 houses (Breteau index), indicating an elevated risk for DENV transmission (5). Among the 1,908 water containers surveyed, 25 (1.3\%) were colonized (container index). Twelve (11.4\%) houses had $\geq 1$ colonized container (house index). The most common types of infested containers were buckets and plastic containers other than buckets, representing $40 \%$ and $16 \%$ of all infested containers, respectively.

\section{Discussion}

Since 2005, two confirmed dengue outbreaks in Mexico that affected the U.S.-Mexico border region have been reported. During 2005 in Brownsville, Texas, 25 dengue cases (including three locally acquired cases) were reported ( 6 ), and during 2013 in Cameron, Hidalgo, and Willacy counties (Texas), 53 cases (26 locally acquired) were reported (7). These outbreaks highlight risk for local DENV transmission in the United 
States, particularly during epidemics in northern Mexico. The number of DENV infections among persons residing near the border is likely higher than the number of reported cases: a 2004 serosurvey of 300 residents of southern Texas identified recent infection among $2 \%$ and past infection among $40 \%(8)$.

This outbreak of travel-associated dengue in Arizona was associated with a ninefold increase in cases compared with any previous single year. Although no locally acquired DENV infections were identified in Arizona during 2014, the increase in travel-associated cases is noteworthy, because established populations of Ae. aegypti put Yuma County at risk for local DENV transmission. The Breteau and household indices identified in the household cluster investigation are above WHO thresholds of 20\% and 5\%, respectively, indicating that Yuma County has an elevated risk for local DENV transmission (5). However, such indices were established in areas with endemic dengue, where human-mosquito interaction might exceed that in southern Arizona (9). During a 1999 dengue outbreak in Texas, although Ae. aegypti mosquito densities were higher in Texas than in Mexico, DENV transmission was less common because of environmental and behavioral factors (e.g., air conditioning use) that limit human-mosquito interaction (9). The Arizona-Sonora border region might have similar cross-border differences in environmental factors that limit local DENV transmission, which might explain the absence of identified local DENV infections in Yuma County during 2014, despite active case finding. Alternatively, travel frequency to Mexico might have obscured infection within Yuma County, with locally acquired infections misclassified as travel-associated.

Although the proportion of reported patients with DHF was lower in Yuma County than in San Luis Río Colorado, a higher proportion of patients in Yuma County (53\%) were hospitalized than in San Luis Río Colorado (21\%). This might be because clinicians in Yuma County had a lower threshold for admitting dengue patients to the hospital. In a survey of 197 health care providers in Arizona, lack of confidence to treat mild dengue and severe dengue was reported by $58 \%$ and $73 \%$ of respondents, respectively (Arizona Department of Health Services, unpublished data, 2014). Dengue patients who do not have warning signs (abdominal pain or tenderness, persistent vomiting, clinical fluid accumulation [ascites or pleural effusion], mucosal bleeding, lethargy or restlessness, liver enlargement $>2 \mathrm{~cm}$, or increase in hematocrit concurrent with rapid decrease in platelet count) ${ }^{\S}$ or high-risk comorbid conditions can be monitored as outpatients with follow-up (1). Clinicians can improve their knowledge of dengue clinical case management through a course available online.9

\footnotetext{
${ }^{\$}$ http://www.cdc.gov/dengue/clinicalLab/caseDef.html.

' http://www.cdc.gov/dengue/training/cme.html.
}

\section{Summary}

What is already known about this topic?

Dengue is an acute febrile illness caused by any of four dengue virus-types (DENV-1-4), which are transmitted by mosquitos of the genus Aedes and are endemic throughout the tropics and subtropics. During 2010, an estimated 390 million DENV infections and 96 million clinically apparent cases occurred worldwide. Since 2005, two reported dengue outbreaks in Mexico that spread to Texas along the U.S.-Mexico border region have been reported.

What is added by this report?

During September-December 2014, while a dengue outbreak was ongoing in Sonora, Mexico, 93 travel-associated dengue cases were reported in Arizona; $75 \%$ of cases were among residents of Yuma County, which borders San Luis Río Colorado, Sonora, Mexico. Among 194 persons in Yuma County surveyed, 152 (78\%) reported travelling to Mexico $\geq 1$ time/month, and elevated Breteau, household, and container Aedes mosquito density indices were consistent with an increased risk for DENV transmission, demonstrating that Yuma County is at risk for local DENV transmission.

What are the implications for public health practice?

Sharing surveillance data among local, state, and federal public health workers in the United States and Mexico can enable timely detection of binational disease outbreaks. Border communities with Aedes mosquitos are at risk for local transmission of DENV, chikungunya virus, and Zika virus infections. Public health messaging to the community should continue to emphasize the importance of mosquito control and avoidance, and conduct surveillance for Aedes mosquitoes to identify areas at risk and prepare response plans for imported and locally acquired DENV, chikungunya virus, and Zika virus infections.

The findings in this report are subject to at least three limitations. First, differences in dengue surveillance protocols between San Luis Río Colorado and Yuma County precluded direct statistical case data comparisons. Second, because not all households or household residents were included in the household cluster investigation, locally acquired dengue cases might not have been identified. Finally, because the investigation was conducted during the colder months of December-January, which reduces the ability of Ae. aegypti to survive and reproduce (10), measured mosquito indices and the associated risk for DENV transmission were likely underestimated compared with those during the outbreak peak.

The collaboration among public health authorities in Mexico and the United States and between local, state, and federal health officials facilitated the sharing of clinical and epidemiologic data and enabled active case finding; ongoing sharing of surveillance data might facilitate timely detection of cross-border outbreaks. An increase in the number of dengue cases in communities near international borders can 
prompt public health officials to increase surveillance efforts and enhance public education regarding mosquito control and avoidance. Notifying health care providers about dengue cases through channels such as the Health Alert Network can raise the index of suspicion for dengue and lower the testing threshold. Public health messaging to the community should emphasize the importance of regularly emptying or disposing of water containers that can serve as mosquito breeding sites; covering or using insecticides in water containers that cannot be emptied; and avoiding mosquito bites by applying mosquito repellent, installing and maintaining window screens, using air conditioning when inside, and wearing long clothing. Public health officials should conduct surveillance for Aedes mosquitos to identify areas at risk and prepare response plans for imported and locally acquired dengue cases (1). Health care providers should solicit travel histories from patients with febrile illnesses, request correct molecular and serologic dengue diagnostic testing for persons with compatible symptoms (1), and report suspected cases to public health authorities. Because Aedes mosquitoes also transmit chikungunya and Zika viruses, there is risk for local transmission of these pathogens and a need for vector control and mosquito bite prevention strategies in the border region.

\footnotetext{
${ }^{1}$ Arizona Department of Health Services; ${ }^{2}$ Epidemic Intelligence Service, Division of Scientific Education and Professional Development, CDC; ${ }^{3}$ Yuma County Public Health Services District, Yuma, Arizona, ${ }^{4}$ Field Services Branch, Division of State and Local Readiness, CDC; ${ }^{5}$ Sonora Department of Health, Hermosillo, Sonora, Mexico; ${ }^{6}$ San Luis Río Colorado Hospital, San Luis Río Colorado, Sonora, Mexico; ${ }^{7}$ Division of Vector-Borne Diseases, National Center for Emerging and Zoonotic Infectious Diseases, CDC, San Juan, Puerto Rico; ${ }^{8}$ University of Arizona; ${ }^{9}$ National Center for Atmospheric Research, Boulder, Colorado; ${ }^{10}$ Division of Global Migration and Quarantine, National Center for Emerging and Zoonotic Infectious Diseases, CDC, San Diego, California.
}

Corresponding author: Jefferson Jones, jjones10@cdc.gov, 602-376-8251.

\section{Dengue Investigative Team}

Irene Ruberto, PhD, Arizona Department of Health Services; Marilee Kellis, Arizona Department of Health Services; Cimma Sefat, Arizona Department of Health Services; Hayley Yaglom, MPH, Arizona Department of Health Services; Rosa Lira, Arizona Department of Health Services; Zimy Wansaula, MD, Arizona Department of Health Services; Felicia McLean, Arizona Department of Health Services; Candice Robinson, MD, Arizona Department of Health Services; Joli Weiss, PhD, Arizona Department of Health Services; Clarisse A. Tsang, MPH, Arizona Department of Health Services; Christine Wampler, Arizona Department of Health Services; Catherine Golenko, MPH, Arizona Department of Health Services; Joey Martinez, Yuma County Public Health Services District, Arizona; Rachael Baker, Yuma County Public Health
Services District, Arizona; Richard Cumming, Yuma County Public Health Services District, Arizona; Emily Pieracci, DVM, Division of Vector-Borne Diseases, National Center for Emerging and Zoonotic Infections Diseases, CDC, San Juan, Puerto Rico; Daniel Williamson, University of Arizona, Kaite Shinaberry, University of Arizona; Eileen Jeffrey, University of Arizona; Kathleen Walker, PhD, University of Arizona; Sonia Montiel, Division of Global Migration and Quarantine, National Center for Emerging and Zoonotic Infectious Diseases, CDC; Carla DeSisto, MPH, Division of Global Migration and Quarantine, National Center for Emerging and Zoonotic Infectious Diseases, CDC; Maureen Fonseca-Ford, MPH, Division of Global Migration and Quarantine, National Center for Emerging and Zoonotic Infectious Diseases, CDC; Alfonso Rodriguez, DVM, Division of Global Migration and Quarantine, National Center for Emerging and Zoonotic Infectious Diseases, CDC; Meredith Binford, Epidemiology Elective Program, CDC; Stuart Cofield, Epidemiology Elective Program, CDC; (all of these individuals meet collaborator criteria).

\section{References}

1. World Health Organization. Dengue: guidelines for diagnosis, treatment, prevention and control: new edition. Geneva, Switzerland: World Health Organization; 2009.

2. Bhatt S, Gething PW, Brady OJ, et al. The global distribution and burden of dengue. Nature 2013;496:504-7. http://dx.doi.org/10.1038/ nature 12060

3. Instituto de Diagnóstico y Referrencia Epiedemiológico. Lineamientos para la Vigilancia Epidemiolóica de Dengue por Laboratorio. [Guidelines for laboratory surveillance and epidemiology of dengue]. Mexico City: Instituto de Diagnóstico y Referrencia Epiedemiológico; 2015. http:// www.indre.salud.gob.mx/sites/indre/descargas/pdf/Lineamientos/ lineamientos_para_la_vigilancia_de_dengue.pdf

4. World Health Organization. Dengue haemorrhagic fever: diagnosis, treatment, prevention, and control. 2nd ed. Geneva, Switzerland: World Health Organization; 1997.

5. World Health Organization. Guidelines for dengue surveillance and mosquito control. 2nd ed. Manila, Phillippines: World Health Organization Regional Office for the Western Pacific, Manila; 2003.

6. Ramos MM, Mohammed H, Zielinski-Gutierrez E, et al.; Dengue Serosurvey Working Group. Epidemic dengue and dengue hemorrhagic fever at the Texas-Mexico border: results of a household-based seroepidemiologic survey, December 2005. Am J Trop Med Hyg 2008;78:364-9.

7. Thomas DL, Santiago GA, Abeyta R, et al. Reemergence of dengue in southern Texas, 2013. Emerg Infect Dis 2016. http://dx.doi. org/10.3201/eid2206.152000

8. Brunkard JM, Robles López JL, Ramirez J, et al. Dengue fever seroprevalence and risk factors, Texas-Mexico border, 2004. Emerg Infect Dis 2007;13:1477-83. http://dx.doi.org/10.3201/eid1310.061586

9. Reiter P, Lathrop S, Bunning M, et al. Texas lifestyle limits transmission of dengue virus. Emerg Infect Dis 2003;9:86-9. http://dx.doi.org/10.3201/ eid0901.020220

10. Liu-Helmersson J, Stenlund H, Wilder-Smith A, Rocklöv J. Vectorial capacity of Aedes aegypti: effects of temperature and implications for global dengue epidemic potential. PLoS One 2014;9:e89783. http:// dx.doi.org/10.1371/journal.pone.0089783 